\title{
Spectral correlations: understanding oscillatory contributions
}

\author{
B. Mehlig ${ }^{1}$ and M. Wilkinson ${ }^{2}$ \\ ${ }^{1}$ Max-Planck-Institut für Physik komplexer Systeme, Nöthnitzer Str. 38, 01187 Dresden, Germany \\ ${ }^{2}$ Department of Physics and Applied Physics, University of Strathclyde, Glasgow, Scotland, UK
}

(August 2, 2006)

\begin{abstract}
We give a transparent derivation of a relation obtained using a supersymmetric non-linear sigma model by Andreev and Altshuler [Phys. Rev. Lett. 72, 902, (1995)], which connects smooth and oscillatory components of spectral correlation functions. We show that their result is not specific to random matrix theory. Also, we show that despite an apparent contradiction, the results obtained using their formula are consistent with earlier perspectives on random matrix models. In particular, the concept of resurgence is not required.
\end{abstract}

Spectral correlations of complex quantum systems, such as disordered metals and classically chaotic quantum systems, are known to be nearly universal. For small ranges of energy they are well approximated by the Gaussian invariant ensembles of random matrix theory (GXE, where $\mathrm{X}=\mathrm{O}, \mathrm{U}$ or $\mathrm{S}$ stands for orthogonal, unitary and symplectic invariance) [1],2]. Deviations from GXE behaviour at larger energy scales may be consistently incorporated using semiclassical or perturbative approaches [3 5. An interesting development in this field was a paper by Andreev and Altshuler (AA) [6], who introduced a relation which suggests a degree of non-universality in short ranged spectral correlations. Their calculations are based on the non-linear sigma model. Our paper will give a transparent physical insight into their relation.

AA 6 ] consider the spectral two-point correlation function, defined as

$$
R_{\beta}(\epsilon)=\Delta^{2}\langle d(E+\epsilon / 2) d(E-\epsilon / 2)\rangle-1 .
$$

Here $d(E)=\sum_{n} \delta\left(E-E_{n}\right)$ is the density of states, $E_{n}$ are the eigenvalues of a Hamiltonian $\widehat{H}$ and $\Delta(E)=$ $\langle d(E)\rangle^{-1}$ is the mean level spacing which is assumed to be independent of $E$ in the following. The index $\beta=1,2,4$ distinguishes orthogonal, unitary or symplectic symmetry classes respectively.

AA divide Eq. (1) into a smooth and an oscillatory contribution, and propose that (for $\beta=2$ ) these are related as follows

$$
\begin{aligned}
& R_{2}^{\mathrm{av}}(\epsilon) \simeq-\frac{\Delta^{2}}{4 \pi^{2}} \frac{\partial^{2}}{\partial \epsilon^{2}} \log D(\epsilon) \\
& R_{2}^{\mathrm{osc}}(\epsilon) \simeq \frac{1}{2 \pi^{2}} \cos (2 \pi \epsilon / \Delta) D(\epsilon)
\end{aligned}
$$

The relation is exact for the GUE with a suitable choice for $D(\epsilon)$ and generalizations are approximately true for the other ensembles. AA propose that Eqs. (2) and (3) are quite general, and should be used to predict the oscillatory component from the average component. The quantity $D(\epsilon)$ is a spectral determinant.

The AA relation contained in (2) and (3) has attracted considerable attention (see, for example, 79), partly because of the suggestion that it contains information about 'resurgence'. This is to be interpreted in terms of Gutzwiller's 10 relationship between periodic orbits and the density of states: periodic orbits orbits with period $t_{j}$ are associated with oscillations in the density of states of period $\epsilon_{j}=\hbar / t_{j}$. The concept of resurgence is that information about long period orbits is encoded in properties of the short orbits. In this context, $R^{\mathrm{av}}(\epsilon)$ may be derived using Gutzwiller's relation from properties of short orbits, and the AA relation then gives information about fluctuations in the spectrum with wavelength equal to the mean level spacing $\Delta$, corresponding to orbits of period equal to the 'Heisenberg time', $t_{\mathrm{H}}=2 \pi \hbar / \Delta$.

This interpretation of the AA result is at variance with some old ideas about the applicability of random matrix theory. Dyson [11] introduced a Brownian motion model for random matrix theory, giving a Langevin equation of motion for the response of energy levels to a stochastic perturbation. Dyson later suggested [12] that this model may give valuable insights into why random matrix theory applies to generic systems. He considered the dynamics of the energy levels under the effect of a stochastic perturbation, which is large enough to shift energy levels significantly, but which remains small enough to leave other features of the system (such as the classical dynamics) unchanged. Dyson showed that the equations of motion simplify if the discrete Fourier coefficients $a_{k}$ of the level displacements are used as dynamical variables:

$$
a_{k}=\frac{1}{N} \sum_{n=0}^{N-1} \Delta E_{n} \exp (-2 \pi \mathrm{i} k n / N)
$$

where $\Delta E_{n}=E_{n}-n \Delta, N$ is the number of energy levels, and $k$ takes $N$ successive integer values. We will take the maximal $k$ to be $\operatorname{int}(N / 2)$. The long wavelength modes evolve almost independently, with a long relaxation time, which scales as $k^{-1} 12,4$. The short wavelength modes remain strongly coupled. The stochastic perturbation will bring the short wavelength modes into equilibrium, giving statistics of the $a_{k}$ which are identical to the random matrix ensemble. 
These arguments were later supported and extended with the aid of semiclassical estimates of matrix elements [4]. The argument in [12] assumes that the matrix elements of the perturbation are uncorrelated. It was shown that semiclassical estimates are consistent with this hypothesis when considering the stochastic force driving the large $k$ modes. The forces driving the small $k$ modes are modified by the classical dynamics of the system. The resulting picture is that long wavelength fluctuations are non-universal, but that at short wavelengths the excitations of the modes are precisely the same as for the appropriate GXE. In particular, there is no modification of the statistics of the Fourier coefficients $a_{k}$ unless $|k| / N$ is small. This appears to be in contradiction with the AA relations, which suggest that non-universal corrections to the smooth part of the correlation function are echoed by oscillations with large wavenumbers $k$.

One resolution of this contradiction would be that there are previously un-suspected correlations between matrix elements due to 'resurgence', which are not captured by the semiclassical approximations in [4. We will however show that there is no need in invoke this principle. We will first describe a simple derivation of the AA result, and comment on its applicability. We will then describe the Brownian motion model and use it to derive the correlation function $R_{\beta}(\epsilon)$ for the case of a system with diffusive electron motion. In this calculation we assume that the statistics of the Fourier coefficients are unchanged for large $|k|$. The fact that we reproduce existing results verifies that resurgence is not an essential ingredient in their derivation. Finally we will comment on the correlation function for classically chaotic systems.

Our starting point is the following general expression for the correlation function $R_{\beta}(\epsilon)$ :

$$
R_{\beta}(\epsilon)=-1+\Delta \sum_{n=-\infty}^{\infty} p_{\beta}(n, \epsilon)
$$

where $p_{\beta}(n, \epsilon) \mathrm{d} \epsilon$ denotes the probability of finding that the difference between $E_{0}$ and $E_{n}$ is in the interval $[\epsilon, \epsilon+\mathrm{d} \epsilon]$. We will show below that in many cases, the $p_{\beta}(n, \epsilon)$ are well approximated by Gaussians for large $n$. We will estimate the variance $\sigma_{\beta}^{2}(n)$ for different systems.

First, however, we show how the relations (2) and (3) can be derived from (5). We write

$$
R_{\beta}(\epsilon)=R_{\beta}^{\mathrm{av}}(\epsilon)+R_{\beta}^{\mathrm{osc}}(\epsilon) .
$$

Here $R_{\beta}^{\mathrm{av}}(\epsilon)$ is defined as

$$
R_{\beta}^{\mathrm{av}}(\epsilon)=\int_{-\infty}^{\infty} \mathrm{d} \epsilon^{\prime} w\left(\epsilon-\epsilon^{\prime}\right) R_{\beta}\left(\epsilon^{\prime}\right)
$$

where $w(\epsilon)$ is a suitable window function (which could be a Gaussian centred around zero with variance much larger than $\sigma_{\beta}^{2}(n)$ and normalized to $\left.\Delta^{-1}\right) . R_{\beta}^{\text {osc }}(\epsilon)$ is the remaining oscillatory contribution. Using (5) we have, upon expanding the slowly varying window function in (7)

$$
\begin{aligned}
R_{\beta}^{\mathrm{av}}(\epsilon) & =-1+\Delta \sum_{n=-\infty}^{\infty} \int_{-\infty}^{\infty} \mathrm{d} \epsilon^{\prime} w\left(\epsilon-\epsilon^{\prime}\right) p_{\beta}\left(n, \epsilon^{\prime}\right) \\
& \simeq-1+\Delta \sum_{n=-\infty}^{\infty}\left[w(\epsilon-n \Delta)+\frac{1}{2} w^{\prime \prime}(\epsilon-n \Delta) \sigma_{\beta}^{2}(n)\right] .
\end{aligned}
$$

Assuming that $\sigma_{\beta}^{2}(n)$ is a slowly varying function of $n$, we may approximate the sum over $n$ as an integral and obtain

$$
R_{\beta}^{\mathrm{av}}(\epsilon) \simeq \frac{1}{2 \Delta^{2}} \frac{\partial^{2}}{\partial n^{2}} \sigma_{\beta}^{2}(n), \quad \epsilon=n \Delta .
$$

Consider now the oscillatory contribution $R_{\beta}^{\text {osc }}(\epsilon)$. Again assuming that $\sigma_{\beta}^{2}(n)$ is slowly varying, these can be evaluated from Eqs. (5) using Poisson summation:

$$
R_{\beta}^{\mathrm{osc}}(\epsilon) \simeq \sum_{\mu=1}^{\infty} 2 \cos (2 \pi \mu n) \mathrm{e}^{-(2 \pi \mu)^{2} \sigma_{\beta}^{2}(n) / 2 \Delta^{2}}
$$

This sum is dominated by the $\mu=1$ term. Defining $D(\epsilon)=4 \pi^{2} \exp \left[-2 \pi^{2} \sigma^{2}(n) / \Delta^{2}\right]$ we obtain the relations (2) and (3). We remark that these formulae are valid for any correlation function that can be approximated as a sum of Gaussians, in regions where the variance satisfies $\sigma^{2} \gg \Delta^{2}$, and where $\sigma^{2}$ varies sufficiently slowly as a function of $n$. Relating $D(\epsilon)$ to $\sigma^{2}(n)$ gives a clear insight into its meaning. The relations (2) and (3) may be easily extended by considering higher-order derivatives in (2) and higher Fourier components in (3). Extensions to non Gaussian spacing distributions are possible. Also, we emphasize that these relations are not specific to spectral correlation functions. For example, they are applicable to density correlations in solids: for thermal excitation of phonons the corresponding two-point function is a sum of Gaussians, and $\sigma^{2}(n) \sim \log n$ in $d=2$ dimensions [13].

Next we consider the calculation of $p_{\beta}(n, \epsilon)$ and $\sigma_{\beta}^{2}(n)$ using Dyson's Brownian motion model. In the Brownian motion model, the matrix elements of the Hamiltonian $\widehat{H}$ undergo a diffusive evolution as a function of a fictitious time variable $\tau$. We denote the infinitesimal change of $\widehat{H}$ by $\delta \widehat{H}$. In the GOE case, we have for $n>m$ and $n^{\prime}>m^{\prime}$

$$
\left\langle\delta H_{m n}\right\rangle=0,\left\langle\delta H_{m n} \delta H_{m^{\prime} n^{\prime}}\right\rangle=C_{m n}^{\mathrm{off}} \delta \tau \delta_{n n^{\prime}} \delta_{m m^{\prime}}
$$

(extensions for GUE and GSE are given in [11]). The diagonal elements obey

$$
\left\langle\delta H_{n n}\right\rangle=0,\left\langle\delta H_{m m} \delta H_{n n}\right\rangle=2 \delta \tau \beta^{-1} C_{m n}^{\mathrm{diag}} .
$$

Dyson 11] originally discussed the case where $C_{m n}^{\text {diag }}=$ $\delta_{m n}$ and $C_{m n}^{\text {off }}=1$, for which the statistics of the $E_{n}$ are the same as for the GXE. We will argue below that non-universal deviations from the GXE are encoded in 
$C_{m n}^{\text {diag }}=C_{m-n}^{\text {diag }}\left(\right.$ and $\left.C_{m n}^{\text {off }}=C_{m-n}^{\text {off }}\right)$. Using second order perturbation theory leads to a Langevin equation

$$
\delta E_{n}=\sum_{m \neq n} \frac{\left|\delta H_{m n}\right|^{2}}{E_{n}-E_{m}}+\delta H_{n n}
$$

for the energy level shifts $\delta E_{n}$. Thus

$$
\begin{aligned}
& \left\langle\delta E_{n}\right\rangle=\delta \tau \sum_{m \neq n} \frac{C_{m-n}^{\text {off }}}{E_{m}-E_{n}} \\
& \left\langle\delta E_{m} \delta E_{n}\right\rangle=2 \delta \tau \beta^{-1} C_{m-n}^{\text {diag }}
\end{aligned}
$$

Semiclassical estimates [14 indicate that the $C_{n}^{\text {off }}$ decrease for large values of $n$, i.e. the repulsive interaction is screened at long range. This effect was considered in [15]; for our purposes it is not significant, and we will set $C_{n}^{\text {olf }}=1$ throughout. We now use (4) to obtain the same equations of motion in terms of the Fourier variables $a_{k}$. Using $\delta \Delta E_{n}=\delta E_{n}$, we have

$$
\left\langle\delta a_{k} \delta a_{l}^{*}\right\rangle=2 \delta \tau \beta^{-1} I_{k} \delta_{k l}
$$

where $I_{k}=N^{-1} \sum_{n} C_{n}^{\text {diag }} \exp (-2 \pi \mathrm{i} k n / N)$. The transformation of the drift term is less straightforward. In general the expectation value of $\delta a_{k}$ is a complicated function of all of the $a_{k}$, but in the limit $|k| / N \rightarrow 0$ the equations decouple and obey 11

$$
\left\langle\delta a_{k}\right\rangle=-\frac{2 \pi^{2} k}{N \Delta^{2}} a_{k} \delta \tau .
$$

By solving a Fokker-Planck equation for the real and imaginary parts of $a_{k}$, these are found to have a steady state distribution which is Gaussian. The value of $\left\langle\left|a_{k}\right|^{2}\right\rangle$ can be deduced by requiring that $\left\langle\left|a_{k}(\tau+\delta \tau)\right|^{2}\right\rangle=$ $\left\langle\left|a_{k}(\tau)\right|^{2}\right\rangle$ : writing $a_{k}(\tau+\delta \tau)=a_{k}(\tau)+\delta a_{k}$ and using (16) and (17) we find, provided $|k| / N \ll 1$ :

$$
\left\langle\left|a_{k}\right|^{2}\right\rangle=\frac{N \Delta^{2} I_{k}}{2 \pi^{2} \beta k}
$$

We cannot determine $\left\langle\left|a_{k}\right|^{2}\right\rangle$ for larger values of $|k|$ from this approach. We now make our key assumption, that $I_{k} \sim N^{-1}$ when $|k| / N$ is not small. This corresponds to assuming that there are no short-ranged correlations between the diagonal matrix elements, i.e. $C_{n-m}^{\text {diag }} \sim \delta_{n m}$. Semiclassical arguments which support, but do not prove, this assumption are given in [4]. (The existence of short ranged correlations would represent a type of 'resurgence', in the sense discussed earlier). Under this assumption, the equations of motion of the $a_{k}$ are identical to those for the Brownian motion model describing Gaussian invariant ensembles. We therefore conclude that when $|k| / N$ is not small, the mode intensities $\left\langle\left|a_{k}\right|^{2}\right\rangle$ are identical to those of the Gaussian invariant ensembles.

For large $n$, the level spacings $E_{n}-E_{0}$ are seen to be a sum of many independent random variables and are thus Gaussian distributed

$$
p_{\beta}(n, \epsilon)=\left[2 \pi \sigma_{\beta}^{2}(n)\right]^{-1 / 2} \mathrm{e}^{-(\epsilon-n \Delta)^{2} / 2 \sigma_{\beta}^{2}(n)}
$$

with mean $n \Delta$ and with variance

$$
\sigma_{\beta}^{2}(n)=4 \sum_{k}\left\langle\left|a_{k}\right|^{2}\right\rangle \sin ^{2}\left(\frac{\pi n k}{N}\right) .
$$

Writing $t=t_{\mathrm{H}} k / N$, and $N I_{k}=I(t)$, Eq. (20) becomes

$$
\sigma_{\beta}^{2}(n)=\frac{4 \Delta^{2}}{\beta \pi^{2}} \int_{0}^{t_{\mathrm{H}} / 2} \frac{\mathrm{d} t}{t} J_{\beta}(t) \sin ^{2}\left(\frac{n t \Delta}{2 \hbar}\right)
$$

where $J_{\beta}(t)=I(t)$ for $t \ll 1$, and $J_{\beta}(t)$ takes a universal (but to us, unknown) form when $t$ is not small. For the Gaussian invariant ensembles (where $I(t)=1$ ), the variances clearly grow logarithmically with $n$. They are, asymptotically for large $n$,

$$
\sigma_{\beta}^{2}(n) \simeq \frac{2 \Delta^{2}}{\beta \pi^{2}}\left[\log (2 \pi n)+C_{\beta}\right]
$$

with $C_{1}=-\log \sqrt{2}, C_{2}=0$ and $C_{4}=\log (4 / \pi)$. Using this expression in (2) and (3) gives the correct leading order contributions to $R_{\beta}^{\text {av }}(\epsilon)$ and $R_{\beta}^{\text {osc }}(\epsilon)$ in the limit $\epsilon / \Delta \rightarrow \infty$ (c.f. [2]).

Next we consider how the function $I(t)$ must be modified at small $t$ to take account of classical dynamics. For small values of $|k|$, the amplitude $\delta a_{k}$ can be estimated semiclassically [4]

$$
\delta a_{k} \sim \frac{1}{N} \operatorname{tr}[\delta \widehat{H} \widehat{U}(t)], \quad t=\frac{2 \pi \hbar}{\Delta} \frac{k}{N}=t_{\mathrm{H}} \frac{k}{N}
$$

where $\hat{U}(t)=\exp (-\mathrm{i} \widehat{H} t)$ is the evolution operator. We consider first diffusive systems (electrons in disordered metals), then systems with a chaotic classical limit.

Diffusive systems. In this case we may consider the perturbation $\delta \widehat{H}$ to be uncorrelated random changes of the site energies $V_{\mathbf{n}}$ in an Anderson tight-binding model 15

$$
\delta H=\sum_{\mathbf{n}} \delta V_{\mathbf{n}} \widehat{P}_{\mathbf{n}}, \quad\left\langle\delta V_{\mathbf{n}} \delta V_{\mathbf{n}^{\prime}}\right\rangle=\delta_{\mathbf{n}, \mathbf{n}^{\prime}}
$$

where $\widehat{P}_{\mathbf{n}}$ is the projection for locating an electron on lattice site $\mathbf{n}$. Using the semiclassical approximation (23), $I(t)$ is seen to to be proportional to the probability of returning to the original site after time $t$. Normalising so that $I(t)$ approaches unity for large $t$, we have

$$
I(t)=\sum_{\nu=0}^{\infty} \mathrm{e}^{-D k_{\nu}^{2} t}
$$

where the sum is over the eigenmodes of the Helmholtz equation $\left(\nabla^{2}+k_{\nu}^{2}\right) \psi_{\nu}(\mathbf{r})=0$ with Neumann boundary conditions. In a quasi-one dimensional system, $k_{\nu}=$ $\pi \nu / L$. In this case we obtain from Eq. (21) 
$\sigma_{\beta}^{2}(n)=\frac{2 \Delta^{2}}{\beta \pi^{2}}\left[\log (2 \pi n)+C_{\beta}+\frac{1}{2} \sum_{\nu=1}^{\infty} \log \left(1+\frac{n^{2}}{g^{2} \nu^{4}}\right)\right]$

where $g=\pi^{2} \hbar D / L^{2} \Delta$ is a dimensionless conductance.

An alternative way to express the results for $R_{\beta}(\epsilon)$ is in terms of the 'form factor' $K(t)$ which is the Fourier transform of $R_{\beta}(\epsilon)$. As discussed by AA, Eq. (3) gives rise to non-universal structures in the form factor at the Heisenberg time $t_{\mathrm{H}}$. We have verified their existence in numerical simulations: we used an ensemble of complex Hermitian banded random matrices (of dimension $N=1000$ and band width $b=35$ ) modelling a quasi-one dimensional diffusive system. We fitted the dimensionless conductance, using states from the centre of the spectrum (obtaining $g \simeq 2.0$ ). The results are shown in Fig. 11 and are in good agreement with the theoretical predictions, Eqs. (9), (10) and (26).

The theoretical predictions shown in Fig. 1 are precisely equivalent to the AA results (7) and (14) in [6]. We have thus shown that their results are consistent with the much simpler approach to justifying random matrix theory discussed in 12.

Classically chaotic systems. Consider the expression (23) for the fluctuation $\delta a_{k}$ of the Fourier coefficients. If the system has a smooth classical Hamiltonian, this expression will clearly be negligible unless $t$ corresponds approximately with the period of a periodic orbit. Moreover, it was shown in [4] that when the motion is chaotic the $\delta a_{k}$ have statistics corresponding to random matrix theory for large $t$. A simple model capturing the essential features of this case is to replace the lower limit of the integral in (21) with the period $t_{0}$ of the shortest periodic orbit. This gives:

$$
\sigma_{\beta}^{2}(n) \simeq \frac{2 \Delta^{2}}{\beta \pi^{2}}\left[\operatorname{Ci}\left(\frac{2 \pi n t_{0}}{t_{\mathrm{H}}}\right)-\log \left(\frac{t_{0}}{t_{\mathrm{H}}}\right)-\gamma+C_{\beta}\right] .
$$

This model has the feature that $\sigma_{\beta}^{2}(n)$ is finite in the limit $n \rightarrow \infty$, corresponding to the behaviour of Dyson's $\Delta_{3}$ statistic for systems with a smooth classical limit [3]. We note that this implies [using (2) and (3)] that the oscillatory part of the correlation function does not decay to zero. The form factor is seen to have a delta function at the Heisenberg time $t_{\mathrm{H}}$, with a magnitude proportional to $\left(t_{0} / t_{\mathrm{H}}\right)^{4 / \beta}$. This feature has not been remarked upon in earlier papers which have discussed the form factor for chaotic systems [7] 9].

A more precise estimate of $\sigma_{\beta}^{2}(n)$ for specific systems can be obtained using periodic orbit theory, following the approach used in [3]. The conclusions are unchanged: $\sigma_{\beta}^{2}(n)$ remains finite as $n \rightarrow \infty$, and there must exist oscillations in the correlation function which do not decay. One difference is that for large $n$ the $p_{\beta}(n, \epsilon)$ are dominated by the shortest orbits. Because only a finite number of components are significant, the central limit theorem cannot be used to assert that these distributions are Gaussian. Moreover small deviations from a Gaussian distribution can have a large effect on the Fourier transform of the distribution, which determines the magnitude of the oscillations in (10). We infer that the AA relations may need to be modified when applied to the spectra of systems with a smooth classical limit.

Conclusions. We have shown that the AA relations have a simple interpretation, independent of random matrix theory and supersymmetric techniques. We have shown how spectral correlations of diffusive systems are obtained using from Dyson's Brownian motion model, indicating that the AA relation is consistent with this approach.

Acknowledgements. It is a pleasure to acknowledge illuminating discussions with O. Agam. BM was partially supported by the SFB 393. MW was supported by the Max Planck Institute for the Physics of Complex Systems, Dresden, and the EPSRC, grant GR/L02302.

[1] F. Haake, Quantum Signatures of Chaos, Springer, New York (1991).

[2] M. L. Mehta, Random matrices, Academic Press, San Diego (1991).

[3] M. V. Berry, Proc. Roy. Soc. A400, 229-51 (1985).

[4] M. Wilkinson, J. Phys. A21, 1173 (1988).

[5] B. L. Altshuler and B. I. Shklovskii, Sov. Phys. JETP 64, 1 (1986).

[6] A. V. Andreev and B. L. Altshuler, Phys. Rev. Lett. 75, 902 (1995).

[7] O. Agam, B. L. Altshuler and A. V. Andreev, Phys. Rev. Lett. 75, 4389 (1995).

[8] E. Bogomolny and J. P. Keating, Phys. Rev. Lett. 77, 1472 (1996).

[9] R. Prange, Phys. Rev. Lett. 77, 2447 (1996).

[10] M. C. Gutzwiller, Chaos in Classical and Quantum Mechanics, Springer, New York (1990).

[11] F. J. Dyson, J. Math. Phys., 3, 1191 (1962).

[12] F. J. Dyson, J. Math. Phys., 13, 90 (1972).

[13] R. Peierls, Surprises in Theoretical Physics, Princeton University Press, Princeton (1979).

[14] M. Wilkinson, J. Phys., A20, 2415 (1987).

[15] J. T. Chalker, I. V. Lerner and R. A. Smith, Phys. Rev. Lett. 77, 554 (1996).

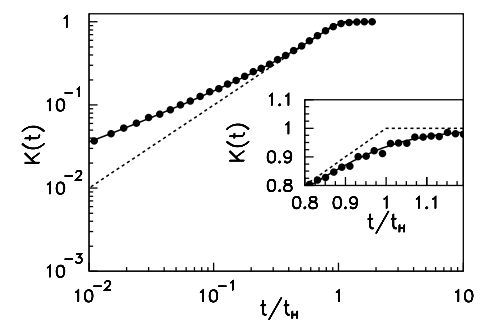

FIG. 1. The form factor $K(t)$ for a quasi-one dimensional diffusive system. Numerical simulations for banded random matrices $(\bullet)$, are compared with theoretical results $(-)$ according to Eqs. (9), (10) and (26). The GUE result is also shown $(---)$. 